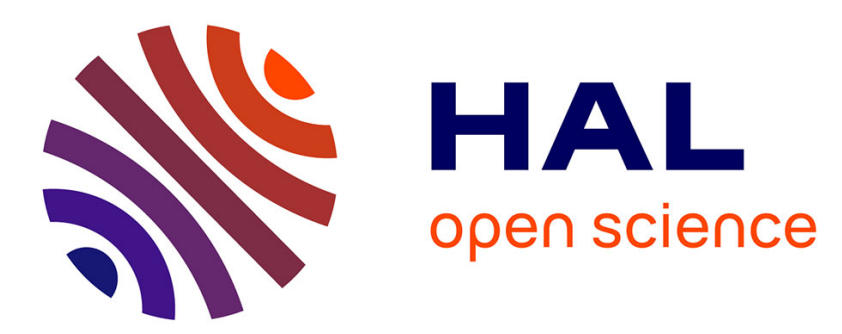

\title{
Using Belief Theory to formalize the agent behavior : application to the simulation of avian flu propagation
}

Patrick Taillandier, Edouard Amouroux, Duc-An Vo, Ana-Maria Olteanu

\section{To cite this version:}

Patrick Taillandier, Edouard Amouroux, Duc-An Vo, Ana-Maria Olteanu. Using Belief Theory to formalize the agent behavior: application to the simulation of avian flu propagation. Pacific Rim workshop on Agent-based modeling and simulation of Complex Systems, 2010, Kolkata, India. pp.575587, 10.1007/978-3-642-25920-3_42. hal-00691406

\section{HAL Id: hal-00691406 https://hal.science/hal-00691406}

Submitted on 27 Apr 2012

HAL is a multi-disciplinary open access archive for the deposit and dissemination of scientific research documents, whether they are published or not. The documents may come from teaching and research institutions in France or abroad, or from public or private research centers.

$$
\text { Copyright }
$$

L'archive ouverte pluridisciplinaire HAL, est destinée au dépôt et à la diffusion de documents scientifiques de niveau recherche, publiés ou non, émanant des établissements d'enseignement et de recherche français ou étrangers, des laboratoires publics ou privés. 


\title{
Using Belief Theory to formalize the agent behavior: application to the simulation of avian flu propagation
}

\author{
Patrick Taillandier ${ }^{1,2}$, Edouard Amouroux ${ }^{1,2}$, Duc An $\mathrm{Vo}^{1,2}$, Ana-Maria Olteanu- \\ Raimond $^{3}$ \\ ${ }^{1}$ IRD, UMI UMMISCO 209, \\ 32 avenue Henri Varagnat, 93143 Bondy, France \\ ${ }^{2}$ IFI, MSI, UMI 209, \\ ngo 42 Ta Quang Buu, Ha Noi, Viet Nam \\ ${ }^{3}$ France Telecom, SENSE Laboratory \\ 38-40, rue du Général Leclerc, 92794 Issy les Moulineaux, France \\ patrick.taillandier@gmail.com, edouard.amouroux@ird.fr, voducanvn@yahoo.com \\ anamaria.raimond@yahoo.fr
}

\begin{abstract}
Multi-agent simulations are powerful tools to study complex systems. However, a major difficulty raised by these simulations concerns the design of the agent behavior. Indeed, when the agent behavior is lead by many conflicting criteria (needs and desires), its definition is very complex. In order to address this issue, we propose to use the belief theory to formalize the agent behavior. This formal theory allows to manage the criteria incompleteness, uncertainty and imprecision. The formalism proposed divides the decision making process in three steps: the first one consists in computing the basic belief masses of each criterion; the second one in merging these belief masses; and the last one in making a decision from the merged belief masses. An application of the approach is proposed in the context of a model dedicated to the study of the avian flu propagation.
\end{abstract}

Keywords: multi-agent simulation, agent behavior formalization, belief theory, avian flu propagation.

\section{Introduction}

Agent-based simulations are now widely used to study complex systems. However, the problem of the agent design is still an open issue. Indeed, designing realistic agents is a complex task, in particular when their behavior is lead by many conflicting needs and desires. A reason of this complexity comes from the lack of practicable formalisms to define the agent decision-making process. In consequence, most of modern models still use ad hoc formalisms to represent the agent behaviors.

In this paper, we propose a new approach to formalize the behavior of agents: this one is based on the belief theory. This theory allows to formalize the reasoning. It can be used to make a decision between several alternatives according to a set of criteria. An advantage of this theory is that it allows to make decision even with 
incompleteness, uncertainly and imprecision, which is particularly interesting in the simulation context.

The paper is organized as follows. In Section 2, the general context of our work is introduced, in particular the problem of the agent behavior formalization. Section 3 is devoted to the presentation of the belief theory and its application for agent behavior design. Section 4 describes an application of our formalism to define the behavior of poultry flocks in the context of a study of the avian flu propagation. Finally, Section 5 concludes and presents the perspectives of this work.

\section{Context}

\subsection{Formalisms to represent the agent behavior}

In this paper, we are interested in the formalisms used to represent the agent behavior. If many formalisms were defined in the multi-agent community (final state machine, BDI [1], motivational [2], etc.), these ones are not of much use for agentbased simulations. A reason is their inadequacy to the simulation context: a formalism, to be used in simulation, has to allow thousands of agents to make a decision from many criteria in a short amount of time. Thus, the formalisms, such as BDI, that are designed for cognitive agents, rather than reactive ones, are usually not usable for multi-agent simulation. Formalisms such as final state machine can be used for simple agents, but its representation capability is fairly limited.

\subsection{Agent behavior as a multi-criteria decision making problem}

We propose to formulate the behavior of the agents as a multi-criteria decision making problem: at each step of the simulation, the agent has to make a decision: which action to apply? The action choice will be guided by the needs and desires of the agent. We propose to formulate these needs and desires as a set of criteria. Thus, the agent behavior consists in choosing, according to a set of criteria, the most pertinent action.

In the literature, several approaches were proposed to solve this type of multicriteria decision-making problems.

A first family of approaches, called partial aggregation approaches, consists in comparing the different possible decisions per pair by the mean of outranking relations $[3,4]$.

Another family of approaches, called complete aggregation approaches, consists in aggregating all criteria in a single criterion (utility function), which is then used to make the decision $[5,6]$.

A last family of approaches, which is highly interactive, consists in devising a preliminary solution and comparing it with other possible solutions to determine the best one $[7,8]$. 
Partial aggregation approaches allow to address the problem of criterion incompatibility but lack of clarity compare to complete aggregation approaches [9].

The approach we are interested in belongs to the complete aggregation approaches. It inherits from the signal detection theory [10] and is built on the belief theory. In the next section, we describe this approach and its application for the agent behavior design.

\section{Use of the belief theory to design the agent behavior}

\subsection{Multi-criteria decision making using the belief theory}

\section{Generality}

The belief theory, also called Dempster-Shafer theory, was proposed by Shafer in 1976 [11]. It is based on the Theory of Evidence introduced by Dempster [12], which concerns the lower and upper probability distributions. It allows to manage incompleteness, uncertainty and imprecision of data. It has been used with success for many applications (e.g. [13, 14, 15]).

The belief theory first defines a frame of discernment, noted $\Theta$. It is composed of a finite set of hypotheses corresponding to the potential solutions of the considered problem.

$$
\Theta=\left\{H_{1}, H_{2}, \ldots, H_{N}\right\}
$$

From this frame of discernment, let us define the set of all possible assumptions, noted $2^{\Theta}$ :

$$
2^{\Theta}=\left\{\varnothing,\left\{H_{1}\right\},\left\{H_{2}\right\}, \ldots,\left\{H_{1}, H_{2}\right\}, \ldots, \Theta\right\}
$$

Each set $\left\{H_{i}, \ldots, H_{j}\right\}$ represents the proposition that the solution of the problem is one of the hypotheses of this set.

The belief theory is based on the basic belief assignment, i.e. a function that assigns to a proposition $\mathrm{P}$, with $\mathrm{P} \in 2^{\Theta}$, a value named the basic belief mass ( $\mathrm{bbm}$, noted $m_{j}(P)$. It represents how much a criterion $\mathrm{j}$-called source of informationsupports the proposition $\mathrm{P}$. The $b b m$ is ranged between 0 and 1 and is defined as follows:

$$
\sum_{P \in 2^{\Theta}} m_{j}(P)=1
$$

\section{Decision making approach}

In our agent behavior context, each hypothesis represents the fact that an action of the set of actions $A$ is the best one. For example: " $\left\{H_{l}\right\}$ : the best action of $A$ is $a_{1}$ ", " $\left\{H_{2}\right\}$ : the best action of $A$ is $a_{2}$ ", " $\left\{H_{1}, H_{2}\right\}$ : the best action of $A$ can be either $a_{1}$ or $a_{2}$ ", etc.

The decision making approach is composed of four steps. 


\section{Step 1}

This first step consists in initializing the basic belief masses. For this step, we propose to use the works of Appriou [16]. He proposed to "specialize" the criteria for one hypothesis of the discernment frame. Thus, the criteria give one's opinion only in favor of a hypothesis, in disfavor of it or do not give their opinion. For each hypothesis $H_{i}$ of $\Theta$, a subset $S^{i}$ of $2^{\Theta}$ is defined:

$$
S i=\{\{H i\},\{\neg H i\}, \Theta\}
$$

- $\left\{H_{i}\right\}$ : this proposition means that the hypothesis $H_{i}$ is true.

- $\left\{\neg H_{i}\right\}=\Theta-\left\{H_{i}\right\}$ : this proposition means that the hypothesis $H_{i}$ is false.

- $\Theta$. this proposition means the ignorance (i.e. every hypotheses can be true).

Thus, the initialization of the basic belief masses consists in computing, for each criterion $j$ and for each hypothesis $H_{i}$ of $\Theta$, the basic belief masses $m_{j}^{H_{i}}\left(\left\{H_{i}\right\}\right)$, $m_{j}^{H_{i}}\left(\left\{\neg H_{i}\right\}\right)$ and $m_{j}^{H_{i}}(\Theta)$.

To compute all the $b b m$, belief functions have to be defined. A belief function is a function that returns a float value between 0 and 1 according to the value of a considered criterion for a given hypothesis. Let $b f$ be a belief functions, $j$ a criterion and $H_{i}$ a decision of $\Theta$. We note $V_{j}^{H_{i}}$ the value of the criterion $j$ for the hypothesis $H_{i}$.

$$
\operatorname{bf}\left(V_{j}^{H_{i}}\right): \Re \rightarrow[0,1]
$$

Examples of belief functions are given Sections 4.2.

\section{Step 2}

This step consists in combining criteria with each other. We propose to use the conjunctive operator introduced in [17] to provide a combined $\mathrm{bbm}$ synthesizing the knowledge from the different criteria. Let us consider two criteria $C_{1}$ and $C_{2}$. The conjunctive operator is defined as follows:

$$
\forall H_{i} \in \Theta, \forall P \in\left\{\left\{H_{i}\right\},\left\{\neg H_{i}\right\}, \Theta\right\}, m_{C_{1} C_{2}}^{H_{i}}(P)=\sum_{P^{\prime} \cap P^{\prime \prime}=P} m_{C_{1}}^{H_{i}}\left(P^{\prime}\right) \times m_{C_{2}}^{H_{i}}\left(P^{\prime \prime}\right)
$$

The fusion of criteria can introduce a conflict, e.g. when one criterion assigns a bbm not null for the proposition $\left\{H_{i}\right\}$ and another criterion assigns a $b b m$ not null for the proposition $\left\{\neg H_{i}\right\}$ (i.e. when $P^{\prime} \cap P^{\prime \prime}=\phi$ ). This conflict will be taken into account in the decision.

For example, let $\left\{C_{l}, C_{2}\right\}$ be a set of criteria, and $H_{1}$ an hypothesis of $\Theta$. Let the $\mathrm{bbm}$ be defined as follows:

$$
\begin{aligned}
& m_{C_{1}}^{H_{1}}\left(\left\{d_{1}\right\}\right)=0.5, m_{C_{1}}^{H_{1}}\left(\left\{\neg d_{1}\right\}\right)=0.3, m_{C_{1}}^{H_{1}}(\Theta)=0.2 \\
& m_{C_{2}}^{H_{1}}\left(\left\{d_{1}\right\}\right)=0.8, m_{C_{2}}^{H_{1}}\left(\left\{\neg d_{1}\right\}\right)=0, m_{C_{2}}^{H_{1}}(\Theta)=0.2
\end{aligned}
$$


The belief masses resulting after the fusion of $C_{1}$ and $C_{2}$ are equal to:

$$
\begin{aligned}
& m_{C_{1} C_{2}}^{H_{1}}\left(\left\{H_{1}\right\}\right)=m_{C_{1}}^{H_{1}}\left(\left\{H_{1}\right\}\right) \times m_{C_{2}}^{H_{1}}\left(\left\{H_{1}\right\}\right)+m_{C_{1}}^{H_{1}}\left(\left\{H_{1}\right\}\right) \times m_{C_{2}}^{H_{1}}(\Theta)+m_{C_{1}}^{H_{1}}(\Theta) \times m_{C_{2}}^{H_{1}}\left(\left\{H_{1}\right\}\right)=0.66 \\
& m_{C_{1} C_{2}}^{H_{1}}\left(\left\{\neg H_{1}\right\}\right)=m_{C_{1}}^{H_{1}}\left(\left\{\neg H_{1}\right\}\right) \times m_{C_{2}}^{H_{1}}\left(\left\{\neg H_{1}\right\}\right)+m_{C_{1}}^{H_{1}}\left(\left\{\neg H_{1}\right\}\right) \times m_{C_{2}}^{H_{1}}(\Theta)+m_{C_{1}}^{H_{1}}(\Theta) \times m_{C_{2}}^{H_{1}}\left(\left\{\neg H_{1}\right\}\right)=0.06 \\
& m_{C_{1} C_{2}}^{H_{1}}(\Theta)=m_{C_{1}}^{H_{1}}(\Theta) \times m_{C_{2}}^{H_{1}}(\Theta)=0.04 \\
& m_{C_{1} C_{2}}^{H_{1}}(\phi)=m_{C_{1}}^{H_{1}}\left(\left\{H_{1}\right\}\right) \times m_{C_{2}}^{H_{1}}\left(\left\{\neg H_{1}\right\}\right)+m_{C_{1}}^{H_{1}}\left(\left\{\neg H_{1}\right\}\right) \times m_{C_{2}}^{H_{1}}\left(\left\{H_{1}\right\}\right)=0.24
\end{aligned}
$$

This conjunctive operator is commutative and associative. Thus, it is possible to combine the result of a previous fusion with the belief masses of another criterion.

Let $C$ be the criterion set. At the end of this step, for each decision $H_{i}$ of $\Theta$, we obtain the combined belief masses $m_{C}^{H_{i}}\left(\left\{H_{i}\right\}\right), m_{C}^{H_{i}}\left(\left\{\neg H_{i}\right\}\right), m_{C}^{H_{i}}(\Theta)$ and $m_{C}^{H_{i}}(\phi)$.

\section{Step 3}

This step consists in combining hypotheses with each other. This combination is interesting because it allows to take into account in the final ranking, the fact that some criteria reject some hypothesis $\left(\neg H_{i}\right)$.

We propose to use the Dempster operator [12] to compute the belief masses resulting from the combination of two hypotheses $H_{i}$ and $H_{j}$ :

$$
\forall P \in 2^{\Theta}, m_{C}^{H_{i}, H_{j}}(P)=\frac{1}{1-m_{C}^{H_{i}, H_{j}}(\phi)} \sum_{P^{\prime} \cap P^{\prime \prime}=P} m_{C}^{H_{i}, H_{j}}\left(P^{\prime}\right) \times m_{C}^{H_{i}, H_{j}}\left(P^{\prime \prime}\right)
$$

The coefficient $\frac{1}{1-m_{C}^{H_{i}, H_{j}}(\phi)}$ is used to normalize the belief masses obtained. In the case of a total conflict $\left(m_{C}^{H_{i}, H_{j}}(\phi)=1\right)$, no decision can be made.

For example, let $\Theta$ be composed of two hypotheses, $H_{1}$ and $H_{2}\left(\Theta=\left\{H_{1}, H_{2}\right\}\right.$, $\left.\left\{\neg H_{1}\right\}=\left\{H_{2}\right\},\left\{\neg H_{2}\right\}=\left\{H_{1}\right\}\right)$. Let the belief masses be defined as follows:

$$
\begin{aligned}
& m_{C}^{H_{1}}\left(\left\{H_{1}\right\}\right)=0.66, m_{C}^{H_{1}}\left(\left\{\neg H_{1}\right\}\right)=0.06, m_{C}^{H_{1}}(\Theta)=0.04, m_{C}^{H_{1}}(\phi)=0.24 \\
& m_{C}^{H_{2}}\left(\left\{H_{2}\right\}\right)=0, m_{C}^{H_{2}}\left(\left\{\neg H_{2}\right\}\right)=0.5, m_{C}^{H_{2}}(\Theta)=0.5, m_{C}^{H_{2}}(\phi)=0
\end{aligned}
$$

The belief masses resulting from the fusion of $C_{I}$ and $C_{2}$ are equal to:

$$
\begin{aligned}
& m_{C}^{\Theta}(\phi)=m_{C}^{H_{1}}\left(\left\{H_{1}\right\}\right) \times m_{C}^{H_{2}}\left(\left\{H_{2}\right\}\right)+m_{C}^{H_{1}}\left(\left\{H_{1}\right\}\right) \times m_{C}^{H_{2}}(\{\phi\})+m_{C}^{H_{1}}\left(\left\{\neg H_{1}\right\}\right) \times m_{C}^{H_{2}}\left(\left\{\neg H_{2}\right\}\right) \\
& +m_{C}^{H_{1}}\left(\left\{\neg H_{1}\right\}\right) \times m_{C}^{H_{2}}(\{\phi\})+m_{C}^{H_{1}}(\Theta) \times m_{C}^{H_{2}}(\{\phi\})+m_{C}^{H_{1}}(\{\phi\}) \times m_{C}^{H_{2}}\left(\left\{H_{2}\right\}\right) \\
& +m_{C}^{H_{1}}(\{\phi\}) \times m_{C}^{H_{2}}\left(\left\{\neg H_{2}\right\}\right)+m_{C}^{H_{1}}(\{\phi\}) \times m_{C}^{H_{2}}(\{\Theta\})+m_{C}^{H_{1}}(\{\phi\}) \times m_{C}^{H_{2}}(\{\phi\})=0.27 \\
& m_{C}^{\Theta}\left(\left\{H_{1}\right\}\right)=\frac{1}{1-m_{C}^{\Theta}(\phi)} \times\left[m_{C}^{H_{1}}\left(\left\{H_{1}\right\}\right) \times m_{C}^{H_{2}}\left(\left\{\neg H_{2}\right\}\right)+m_{C}^{H_{1}}\left(\left\{H_{1}\right\}\right) \times m_{C}^{H_{2}}(\Theta)\right. \\
& \left.+m_{C}^{H_{1}}(\Theta) \times m_{C}^{H_{2}}\left(\left\{\neg H_{2}\right\}\right)\right]=0.93 \\
& m_{C}^{\Theta}\left(\left\{H_{2}\right\}\right)=\frac{1}{1-m_{C}^{\Theta}(\phi)} \times\left[m_{C}^{H_{1}}\left(\left\{\neg H_{1}\right\}\right) \times m_{C}^{H_{2}}\left(\left\{H_{2}\right\}\right)+m_{C}^{H_{1}}\left(\left\{\neg H_{1}\right\}\right) \times m_{C}^{H_{2}}(\Theta)\right. \\
& \left.+m_{C}^{H_{1}}(\Theta) \times m_{C}^{H_{2}}\left(\left\{H_{2}\right\}\right)\right]=0.04 \\
& m_{C}^{\Theta}(\Theta)=\frac{1}{1-m_{C}^{\Theta}(\phi)} \times\left[m_{C}^{H_{1}}(\Theta) \times m_{C}^{H_{2}}(\Theta)\right]=0.03
\end{aligned}
$$


At the end of this step, a belief mass for each proposition $m_{C}^{\Theta}\left(\left\{H_{1}\right\}\right), m_{C}^{\Theta}\left(\left\{H_{2}\right\}\right), \ldots$, $m_{C}^{\Theta}\left(\left\{H_{1}, H_{2}\right\}\right), \ldots, m_{C}^{\Theta}(\Theta)$ is obtained.

\section{Step 4}

The last step consists in making the decision. We are only interested in the propositions that concern a unique hypothesis (one action) and not a set of hypotheses. Thus, to evaluate each proposition we propose to use the pignistic probability [18].

The pignistic probability of a proposition $A$ is computed by the following formulae:

$$
P(A)=\sum_{A \subseteq B} m(B) \frac{|A|}{|B|}
$$

More a proposition maximizes this probability, more the corresponding hypothesis is true. Thus, the decision making will be based on this probability.

For example, let $\Theta$ be composed of two hypotheses, $H_{1}$ and $H_{2}$ and the belief masses of all the propositions be defined as follows:

$$
m_{C}^{\Theta}\left(\left\{H_{1}\right\}\right)=0.93, m_{C}^{\Theta}\left(\left\{H_{2}\right\}\right)=0.04, m_{C}^{\Theta}(\Theta)=0.03
$$

The resulting pignistic probabilities are:

$$
P\left(\left\{H_{1}\right\}\right)=m_{C}^{\Theta}\left(\left\{H_{1}\right\}\right) \times \frac{1}{1}+m_{C}^{\Theta}(\Theta) \times \frac{1}{2}=0.945 \quad P\left(\left\{H_{2}\right\}\right)=m_{C}^{\Theta}\left(\left\{H_{2}\right\}\right) \times \frac{1}{1}+m_{C}^{\Theta}(\Theta) \times \frac{1}{2}=0.055
$$

Thus, $H_{1}$ has more chance to be true than $H_{2}$.

\subsection{Application of the belief theory to define the agent behavior}

As presented in the previous section, the belief theory allows to make a decision from a set of possible actions according to a set of criteria.

In order to use the belief theory to formalize the behavior of an agent, the modeler has to define several elements:

- A set of criteria that allow to evaluate the different possible actions.

- For each criterion: a belief function for the hypotheses "this action is the best one", "this action is not the best one", "ignorance".

Remark that it is possible to decrease the complexity of the decision making computation by filtering the possible actions: only actions that are pareto-optimal are kept .

For some agents, it will also be possible (and mandatory) to divide the decision making process into several sub-processes. This division can be use to decrease the complexity of the decision process or to use different sets of criteria that will correspond to different steps of reasoning. Indeed, for example, it is possible to divide the decision making process into two steps: the first one consisting of choosing a general objective for the agent (e.g. eating, drinking) and a second consisting in choosing the best place to carry out this objective. Another example is given in Section 4.2. 


\section{Application: model dedicated to the avian flu propagation}

In order to illustrate our agent behavior formalism, we present an application of it for a real model about the H5N1 endemic in North Vietnam. After a brief presentation of a context, we focus on the agent behavior design. A description of the complete model is available in [19].

\subsection{Application context}

H5N1 is still a major threat for both economy and health. It has spread over Asia, Europe and some parts of Africa. Nowadays, the endemic appears to be circumscribed to South East Asia mainly. Nevertheless, the eradication of the virus is far from being achieved. In the North Vietnam context, epidemiologists need to study the mechanisms of its local spread and persistence in the context of semi-industrialized and traditional poultry sectors, in order to limit the impact of the virus. To do so agent-based modeling has been selected for its capabilities of detailed representation, especially concerning the environment, and its flexibility.

Consequently, the purpose of the proposed models is to investigate and evaluate the importance of various factors, including poultry production, environments (especially aquatic ones), topography, etc, on the persistence and spread of H5N1 within a village or a commune in the Red River delta. Specifically, the model is about investigating the relationships between environments (as virus reservoirs) and the traditional or semi-commercial poultry production systems.

The real system modeled here is the H5N1 endemics in the traditional and semiindustrial poultry production sector in the Red River Delta (North Vietnam). We limit the represented system to a village (several prototypes were determined using principle component analysis). Within this system, we focus on farms and poultry flocks. Here, poultry flocks can be duck or chicken. These flocks have various behaviors depending on the types of production. As implied by this description several natural environments are represented: building, inner-village ground, road, rice-field (flooded or dry) and pond. Figure 1 shows a snapshot of the model implemented with the GAMA platform $[20,21]$ 


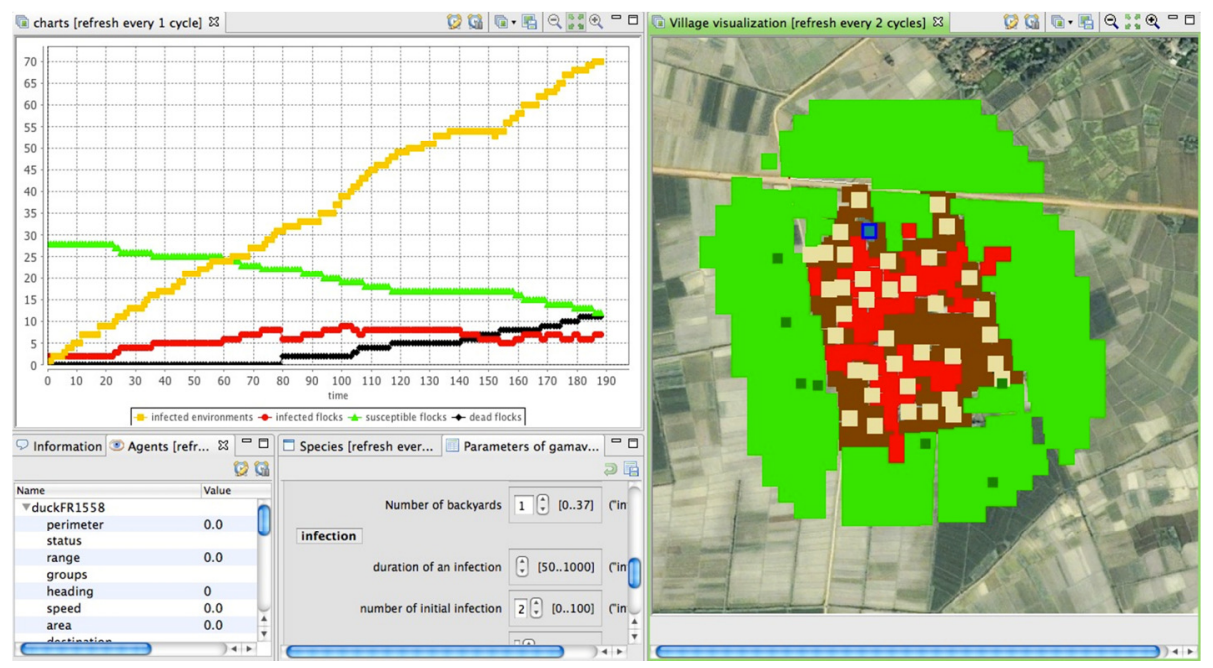

Fig. 1. Model implemented with the GAMA platform [20, 21]

In the next section, we focus on the design of the Flock agent behavior. In particular, we illustrate how the formalism presented in Section 3 is used to design this behavior.

\subsection{Flock behavior design}

In this model, we chose to divide the agent behavior in two steps: first, the agent analyses the best places to eat, to drink and to rest, second, the agent chooses an objective. This one can be to "eat", to "drink", to "go home", "to rest" or "no objective". If no objective is defined, the agent wanders.

The division of the behavior in two steps allows to use different sets of criteria for each step. Moreover, it decreases the computational resources required to make the decision. Indeed, as we are in the context of multi-agent based simulation, the computation complexity of the process is an important factor. The choice to compute first the best places for each activity is mandatory as it impacts the choice of the objective. This allows us to represent the opportunist behavior of a flock, for example: a flock seeing a good place to eat (very close, with a lot of foods and no other flock in the neighborhood) can be tempted to eat at this place even if it is not hungry.

\subsubsection{Place selection}

We defined several criteria to assess the quality of each type of places (eating places, drinking places and resting places), each type of places has the same pool types of criterions. Here the list of criterion along its "belief functions": 
- Distance to the place: this criterion allows to assess the distance between the flock and the candidate places. The belief functions of this criterion are illustrated in Figure 2.

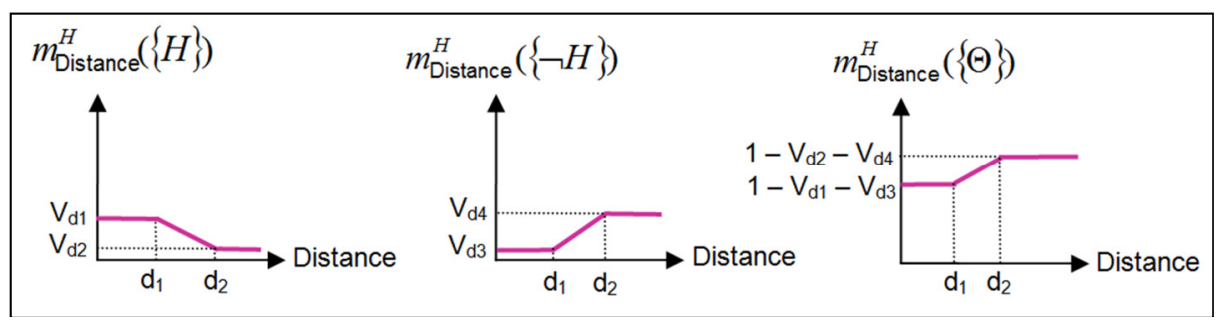

Fig. 2. Belief functions for the distance criterion

- Quantity of resources: this criterion allows to assess the quantity of food contains in the candidate places. Remark that this criterion is not use in the context of the resting place selection. The belief functions of this criterion are presented in Figure 3.

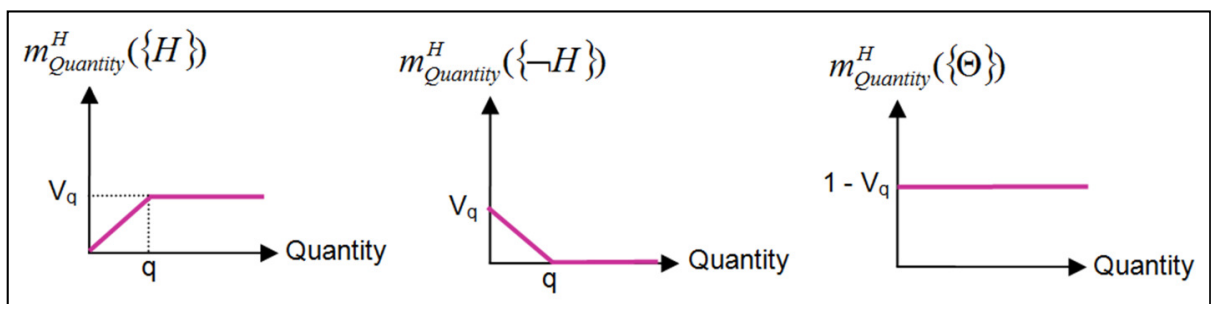

Fig. 3. Belief functions for the quantity of resources criterion

- Quality of the place regarding an objective (i.e. for eating objective, the quality of the place will be the quality of food). This criterion depends on the nature of places: water (W), rice-field (RF), dry-culture (DC) or ground (G). For instance, a flock usually prefers to eat in a rice-field, but it can also eat in a dry-culture field, or at worse directly on the ground or in water. Figures 4, 5 and 6 respectively present the belief functions for the eating, drinking and resting objective. 


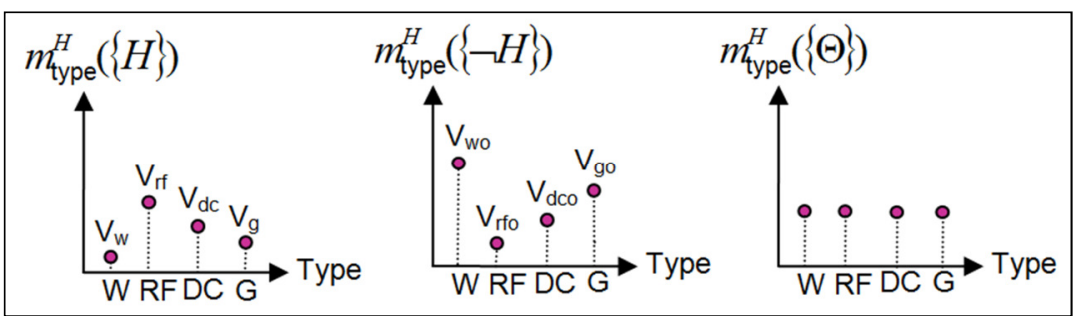

Fig. 4. Belief functions for the quality of the place for eating criterion

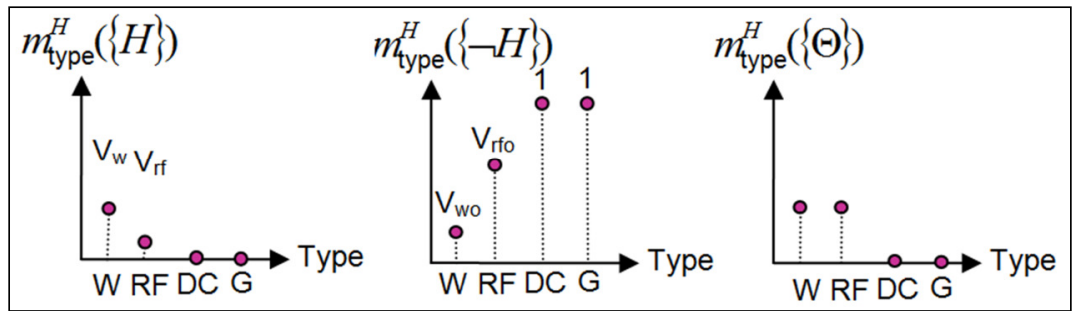

Fig. 5. Belief functions for the quality of the place for drinking criterion

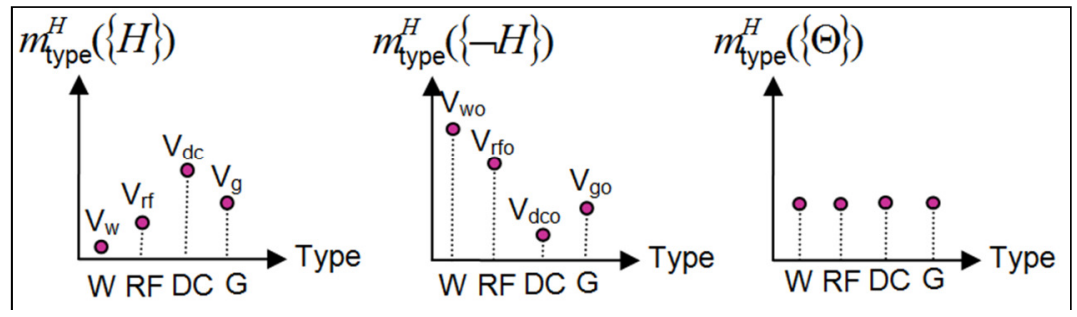

Fig. 6. Belief functions for the quality of the place for resting criterion

- "Agoraphobia": usually, flocks try to avoid to go to place too near of other flocks. Thus, this criterion allows to assess this "agoraphobia". The belief functions of this criterion are illustrated in Figure 7. These functions take as input the number flocks located at distance inferior to $200 \mathrm{~m}$ to the considered flock.

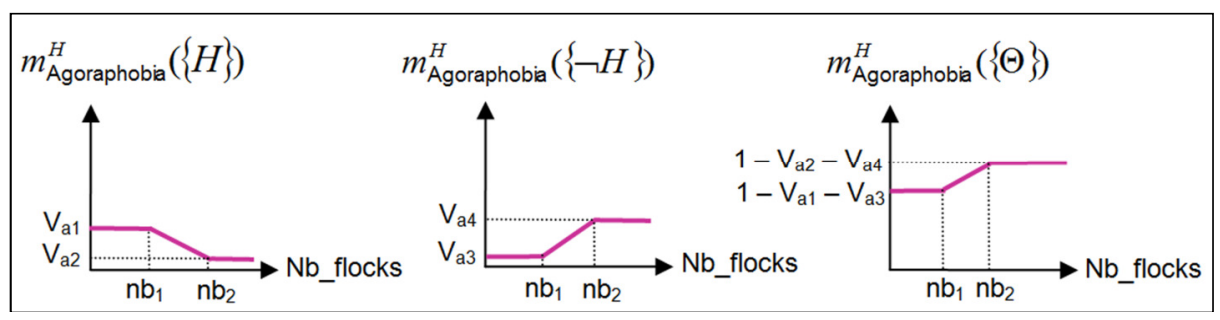

Fig. 7. Belief functions for the agoraphobia criterion 
Once the value of each criterion has been computed, the agent filters the possible candidates in order to keep only the ones belonging to the Pareto-optimal front. Then, the decision making process presented in Section 3 is used to select the most relevant ones (one for each objective: eating, drinking and resting).

\subsubsection{Objective selection}

The second step consists in computing the best objective among the five defined: "eating", "drinking", "resting", "go home" and "no objective". The evaluation of these objectives is based on the internal state of the agents and the time of the day (the environment influence is taken into account during the places selection). The internal state variables related to the objective selection are hunger, thirst, tiredness and "homesickness" levels. They are continuously updated according to the agent's action, for example: if a flock is resting, it will decrease its tiredness and slowly increase the hunger and thirsts levels.

The objective choice is based on several criteria that are described hereafter:

- Time of the day: this criterion allows to specify time intervals for each objective. Indeed, some activities are more likely to be done at specific hours (e.g. going back home at sunset). Figure 9 presents the belief functions of this criterion. These functions take as input the difference between the current time of the day and the time intervals defined for each objective.

- Eating objective: [7am-9am] $\cup[2 \mathrm{pm}-4 \mathrm{pm}]$

○ Drinking objective: [8am-9am] $\cup[2 \mathrm{pm}-5 \mathrm{pm}]$

○ Resting objective: [9am-2pm]

○ Going home objective: [5pm-7pm]

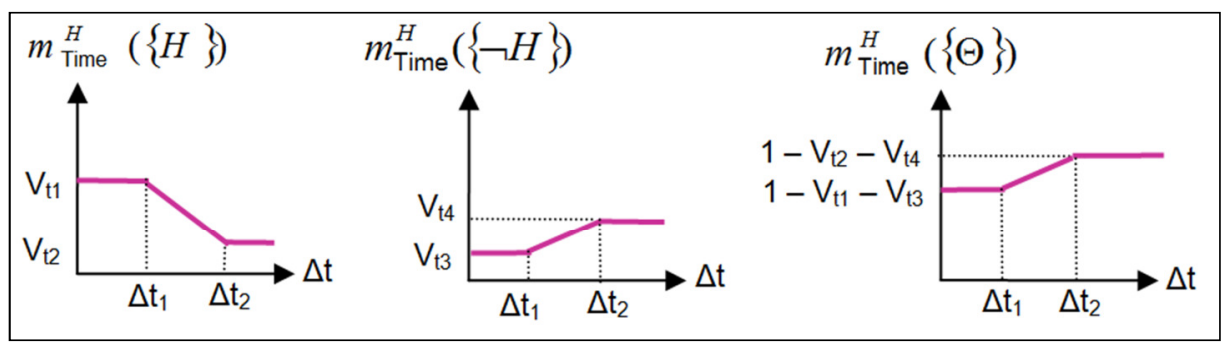

Fig. 8. Belief functions for the time criterion

- Adequacy to the flock needs: this criterion assesses the adequacy between the agent need (internal state) and the different objectives. Figure 9 presents the belief functions of this criterion. These functions take as input:

○ Eating objective: Hunger = max_food_level - current_ food_level

○ Drinking objective: Thirst =max_water_level - current_ water_level

○ Resting objective: Tiredness = time_since_last_rest 
○

Going home: "Home-sickness" = time_since_last_going_home

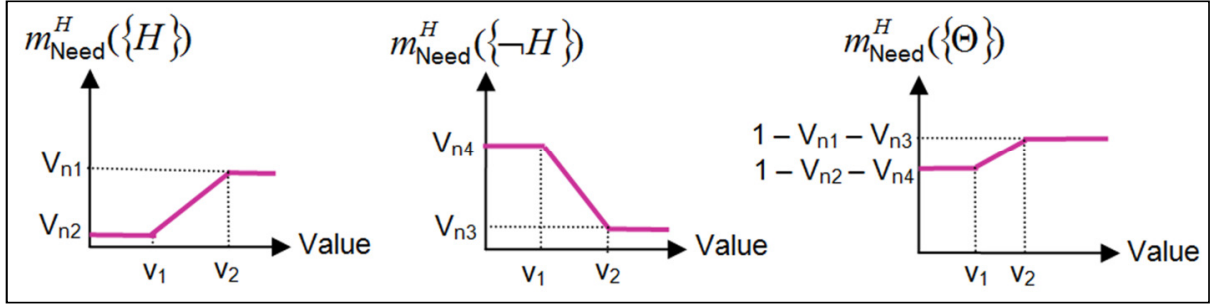

Fig. 9. Belief functions for the need criterion

- Quality of the best selected place: this criterion assesses the quality of the best selected places (the ones selected in Section 4.2.1). The belief functions of this criterion are presented in Figure 10. These functions take as input the value computed at the end of the first step. Concerning the "going home" objective, the value is constant. Indeed, only one place can be chosen for this objective (the flock farm),

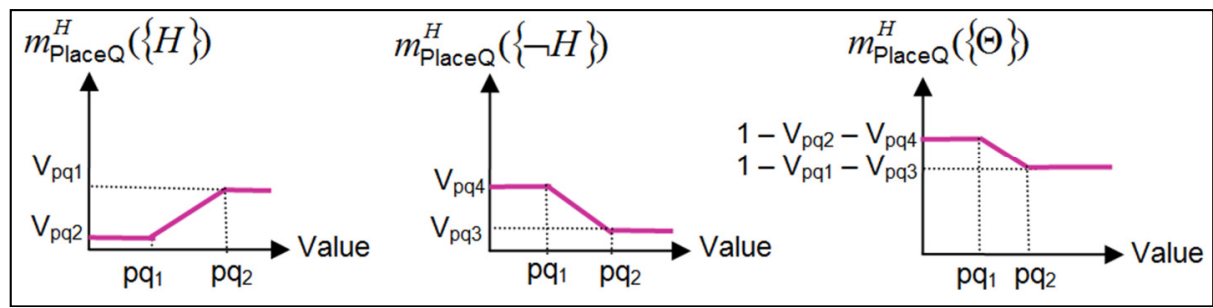

Fig. 10. Belief functions for the place quality criterion

Once the value of each criterion has been computed, the agent uses the decision making process presented in Section 3 to select the most relevant objective (among "eating", "drinking", "resting" and "going home"). If the pignistic probability computed for this objective is lower than a predefined threshold (defined by thematician through interaction with the simulation), no objective is selected and the agent wanders; otherwise, the agent carried out its selected objective.

\section{Conclusion}

In this paper, we proposed to use the belief theory to formalize the agent behavior. We present an application of this formalism for a model dedicated to study H5N1 propagation in North Vietnam. It allowed us to have a precise and realistic representation of flock's behavior while being tunable by field specialist.

In terms of perspective, we want to apply our approach to other models, in particular social model integrating numerous decision criteria. We think that it would be of even more interest to represent more elaborated behavior such has human ones.

A key issue in the use of our formalism concerns the definition of the belief 
functions. In this context, we propose to develop methods to learn directly through a participatory approach. This approach could be based on the one that we proposed in $[22,23]$.

\section{References}

1. Rao, A.S., Georgeff M.P.: Modeling Rational Agents within a BDI-Architecture. In 'Proceedings of the Second International Conference on Principles of Knowledge Representation and Reasoning', pp. 473-484 (1991).

2. Robert, G., Guillot A.: A motivational architecture of action selection for non-player characters in dynamic environments, In 'International Journal of Intelligent Games \& Simulation', vol. 4, pp. 1-12 (2005).

3. Figueira, J., Mousseau, V., Roy, B.: ELECTRE Methods. In: Figueira, J., Greco, S., and Ehrgott, M., (Eds.), Multiple Criteria Decision Analysis: State of the Art Surveys, Springer, New York, 133--162 (2005)

4. Behzadian, M., Kazemzadeh, R., Albadvi, A., Aghdasi, M.: PROMETHEE: A comprehensive literature review on methodologies and applications. European Journal of Operational Research (2009)

5. Geoffrion, A., Dyer, J., Feinberg, A.: An interactive approach for multicriterion optimisation with an application to the operation of an academic department. Manage. Sci., vol. 19 (4), 357--368 (1972)

6. Jacquet-Lagreze, E., Siskos, J.: Assessing a set of additive utility functions for multicriteria decision making, the UTA method. European Journal of Operational Research, vol. 10(2), 151--164 (1982)

7. Benayoun, R., Laritchev, O., de Mongolfier, J., Tegny, J.: Linear programming with multiple objective functions: Step method (stem). Math. Program. 1(3), 366-375 (1971)

8. Ignizio, J.: A review of goal programming: a tool for multi objective analysis. J. Oper. Res. Soc 29(11), - (1978)

9. Ben Mena, S. : Introduction aux méthodes multicritères d'aide à la décision, Biotechnol. Agro. Soc. Environ. 4(2), 83--93 (2000)

10. Marcum, J.: A statistical theory of target detection by pulsed radar. IEEE Trans. Info. Thry. (1960).

11. Shafer, G.: A mathematical theory of evidence. Princeton University Press (1976)

12. Dempster, A.: Upper and lower probabilities induced by multivalued mapping. Annals of Mathematical Statistics, vol. 38, pp. 325-339 (1967)

13. Omrani, H., Ion-Boussier, L., Trigano, P.: A new approach for impacts assessment of urban mobility, in 'WSEAS transaction on Information science and applications' 4(3), pp. 439444 (2007).

14. Olteanu-Raimond, A.M., Mustière, S.: Data matching - a matter of belief, 13th International Symposium on Spatial Data Handling (SDH'08), Montpellier, France, (2008)

15. Taillandier, P., Duchêne, C., Drogoul, A.: Using Belief Theory to Diagnose Control Knowledge Quality. Application to cartographic generalization. In 'IEEE-RIVF', Da Nang City, Viet Nam (2009)

16. Appriou, A.: Probabilité et incertitude en fusion de données multi-senseurs. Revue Scientifique et Technique de la Défense, vol. 1, pp. 27-40 (1991)

17. Smets, P., Kennes, R.: The transferable belief model. Artificial Intelligence vol. 66(2), pp. 191-234 (1994)

18. Smets, P.: Constructing the pignistic probability function in a context of uncertainty. Uncertainty in Artificial Intelligence vol. 5, pp. 29-39 (1990) 
19. Amouroux, E., Gaudou, B., Desvaux, S., Drogoul, A.: O.D.D.: a Promising but Incomplete Formalism For Individual-Based Model Specification. Paper to appear in 'IEEE International Conference on Computing and Telecommunication Technologies' (2010).

20.Amouroux, E., Chu, T-C., Boucher, A., Drogoul, A.: GAMA: an environment for implementing and running spatially explicit multi-agent simulations. International conference PRIMA, Bangkok, (2007)

21. Taillandier, P., Drogoul, A., Vo D.A., Amouroux, A.: GAMA: a simulation platform that integrates geographical information data, agent-based modeling and multi-scale control. In 'The $13^{\text {th }}$ International Conference on Principles and Practices in Multi-Agent Systems (PRIMA)', India. (2010). To appear.

22. Taillandier, P., Buard, E.: Designing Agent Behaviour in Agent-Based Simulation through participatory method. In 'The $12^{\text {th }}$ International Conference on Principles and Practices in Multi-Agent Systems (PRIMA)', Nagoya, Japan. (2009)

23. Taillandier, P., Chu, T.Q.: Using Participatory Paradigm to Learn Human Behaviour. In 'International Conference on Knowledge and Systems Engineering', Ha noi, Viet Nam, pp. 55--60 (2009) 